\title{
The Age of Hesitancy, Recognition, Reinforcement, and Interposition in Cochlear Implant Children in Ahvaz, Iran: A 5-year Study
}

\author{
Nader Saki ${ }^{1}$, Amir Hossein Asadollah Pour ${ }^{1}$, Hasan Abshirini ${ }^{1}$, Soheila Nikakhlagh ${ }^{(10}{ }^{1}$, Majid Karimi ${ }^{1}$, \\ Hossein Bagheripour ${ }^{1}$ and Arash Bayat ${ }^{2,1, *}$ \\ ${ }^{1}$ Hearing Research Center, Imam Khomeini Hospital, Ahvaz Jundishapur University of Medical Sciences, Ahvaz, Iran \\ ${ }^{2}$ Musculoskeletal Rehabilitation Research Center, School of Rehabilitation Sciences, Ahvaz Jundishapur University of Medical Sciences, Ahvaz, Iran \\ "Corresponding author: Hearing Research Center, Imam Khomeini Hospital, Ahvaz Jundishapur University of Medical Sciences, Ahvaz, Iran. Email: arashbayaat@gmail.com
}

Received 2021 January 12; Revised 2021 August 28; Accepted 2021 September 07.

\begin{abstract}
Background: Delay in early detecting of hearing loss (HL) and aural interposition in children have intense impacts on their speech and language expansion, academic progress, social skills, and psychological status.

objectives: The purpose of the current study was to assess the age of hesitancy, recognition, reinforcement, and interposition in children with bilateral severe to deep hearing loss.

Methods: This cross-sectional study was conducted on children who were candidates for cochlear implant surgery at Khuzestan Cochlear Implantation Center, Ahvaz, Iran. The rehabilitative histories and children's medical were utilized to record ages of hesitancy, recognition, reinforcement, and interposition for both groups of "high-risk" and "not-high-risk" children. Paired sample $t$-test was used to evaluate the middle ages of children between the "high-risk" and "not-high risk" groups.

Results: A total of 389 children (205 boys and 184 girls) were included. The mean ages of hearing loss hesitancy, hearing loss recognition, fitting the hearing reinforcement devices, and interposition were $10.7 \pm 7.1,15.75 \pm 9.4,21.81 \pm 8.1$, and $26.32 \pm 9.2$ months, respectively. The average delays between the ages of hesitancy and recognition: $3.74(\mathrm{P}<0.0001)$; recognition and reinforcement: 5.05 ( $\mathrm{P}<0.0001)$; reinforcement and interposition: 2.83 months $(\mathrm{P}<0.0001)$ were obtained. There were no significant statistical differences in terms of four studied ages among the high-risk $(n=129)$ and not-high risk $(n=260)$ children $(P>0.05)$.

Conclusions: The outcomes showed that there is a significant improvement in the average ages of hearing loss hesitancy, recognition, reinforcement, and interposition in Iran. However, there is still an obvious difference between these ages and those recommended by the Joint Committee on Infant Hearing.
\end{abstract}

Keywords: Hesitancy of Hearing Loss, Recognition, Reinforcement, Interposition

\section{Background}

It has been shown that delayed recognition of hearing impairment in children has negative consequences on their speech and language development and social skills. Furthermore, late recognition of HL in children population could limit their academic achievements, reduce their professional and social opportunities, and increase their anxiety level $(1,2)$.

It has been demonstrated that earlier recognition of HL in children, along with early benefiting from auditory amplification and aural rehabilitation services, will improve their speech and language developments proportional to their age (3-7). Yoshinaga-Itano (8) reported that children who were identified before six months old revealed remarkably higher expressive language abilities than children diagnosed after that age, irrespective of the rate of HL or communication modality (9).

Cochlear implantation (CI) is widely known as a standard therapeutic option for patients with intense-to-deep sensorineural hearing loss (SNHL) (10-13). It has been shown that the language skills of children who receive CIs at early ages develop at a similar rate to that of children with hearing impairment. It seems that CI operation in children at a younger age may be associated with enhanced hearing and speech recognition performances, compared to those who are implanted at older ages (14).

\section{Objectives}

The objective of this research was to assess the age of hesitancy, recognition, reinforcement, and interposition in children with bilateral severe to deep HL in children undergoing CI surgery in a CI center in the southwest of Iran. 


\section{Methods}

\subsection{Participants}

The type of research was a cross-sectional study. In this study, 389 prelingually deaf children (205 boys and 184 girls) participated who were approved for CI under the state government CI program from September 2015 to September 2020. These children were recognized within the new-borns hearing screening plan in the southwest of Iran (15). The inclusion criteria entailed being affected by bilateral severe to profound SNHL, having normal temporal bone imaging, complete electrodes insertion into the cochlea, no post-op adverse sequels, and regularly attending pre-and post-operative auditory rehabilitation sessions. Exclusion criteria, overt cognitive delay, or inner ear abnormalities in children were considered. The protocol of the current research was approved by the local Ethics Committee (Registration Number: IR.AJUMS.REC. 1397.958).

\subsection{Procedures}

For the current investigation, the ages of parental hesitancy, recognition, reinforcement, and interposition in hearing-impaired children who were referred to our CI center were recorded in months. The "age of hesitancy" was described as the primary distrust of a child's HL. The "age of recognition" was defined as the first time to pass a hearing examination to confirm the child's HL. The "age of reinforcement" was defined as when a child receives a hearing aid (such as a hearing aid) for the first time and starts using it. Furthermore, the "age of interposition" was defined as the time at which the child began to take auditory training and educational programs. According to the gathered evidence, the course of "hesitancy and recognition", "recognition and reinforcement", and "reinforcement and interposition" were computed.

\subsection{Data Analysis}

All statistical analyses were carried out with SPSS V.24 software. The Kolmogorov-Smirnov test was utilized to evaluate the normality of data distribution for numeric variables. Descriptive statistics were also applied to brief quantitative variables, and stratified variables were reported as percentages. Paired sample $t$-test was used to evaluate the middle ages of hesitancy, recognition, reinforcement, and interposition was investigated among the "high-risk" and "not-high risk" groups. The $\mathrm{P}<0.05$ level was accepted to statistical significance.

\section{Results}

The middle age of hearing loss hesitancy, hearing loss recognition, fitting the hearing reinforcement devices, and interposition was $10.7 \pm 7.1,15.75 \pm 9.4,21.81 \pm 8.1$, and $26.32 \pm 9.2$ months, respectively (Table 1 ). According to our findings, the middle lags among the ages of hesitancy and recognition: $3.74(\mathrm{P}<0.0001)$; recognition and reinforcement: 5.05 ( $\mathrm{P}<0.0001)$; reinforcement and interposition: 4.83 months $(\mathrm{P}<0.001)$ were obtained. These discrepancies were statistically considerable.

In our study, 129 (33.16\%) children were in "high-risk" team. As you can see in Table 1, the averages of 4 studied ages are shown for "high-risk" and "not-high risk" children. Also, our findings showed that there were no considerable statistical differences among both groups ( $\mathrm{P}>$ 0.05). Figure 1 shows the comparison of the ages of children at hesitancy, recognition, reinforcement, and interposition among different studies.

\section{Discussion}

Early recognition and interposition of hearing loss play an essential role in speech and language development. It has been recommended that identification of children suffering from hereditary HL before six months of age and starting hearing rehabilitation will improve their interpersonal and academic skills and improves children's language skills, general language skills, speech intelligibility, vocabulary, and phoneme repertoires, compared to children who have not received their rehabilitation services timely $(15,16)$.

In this research, the middle age of recognition HL was 15.75 months, which is similar to the results of Prendergast et al. (16) and Jafari et al. (17). However, the findings of the present study show obvious differences with the reported cases by Kittrell and Arjmand (18), Lotfi and Jafari (19), and Ozcebe et al. (14) (Table 2).

Our findings demonstrated that the mean age for reinforcement was 21.81 months which is similar to the results of Prendergast et al. (16) and Jafari et al. (17). Our finding, however, indicated lower age at amplification (hearing aid fitting) time compared to those reported by Kittrell and Arjmand (18), and Lotfi and Jafari (19) (Table 2).

In the present study, the middle age of interposition was 26.32 months which is analogous to Prendergast et al.'s (16) findings. In a similar study, Jafari et al. (17) reported that the mean age of interposition for severely hard of hearing children was 22.3 months.

Cochlear implantation has been recommended to enhance relationship proficiency and quality of life in kids with inherent deafness. As a result, these children have 
Saki N et al.

\begin{tabular}{|c|c|c|c|c|}
\hline \multirow{2}{*}{ Group } & \multicolumn{4}{|c|}{ Age (mo) } \\
\hline & Hesitancy & Recognition & Reinforcement & Interposition \\
\hline High-risk children $(n=129)$ & $10.46 \pm 8.65$ & $15.51 \pm 7.91$ & $21.72 \pm 10.05$ & $27.06 \pm 9.33$ \\
\hline $\begin{array}{l}\text { Not high-risk children }(\mathrm{n}= \\
\text { 260) }\end{array}$ & $10.99 \pm 7.63$ & $15.92 \pm 8.37$ & $21.88 \pm 7.43$ & $25.67 \pm 8.82$ \\
\hline $\operatorname{Total}(\mathbf{n}=\mathbf{3 8 9})$ & $10.73 \pm 7.15$ & $15.75 \pm 9.45$ & $21.81 \pm 8.19$ & $26.32 \pm 9.21$ \\
\hline
\end{tabular}

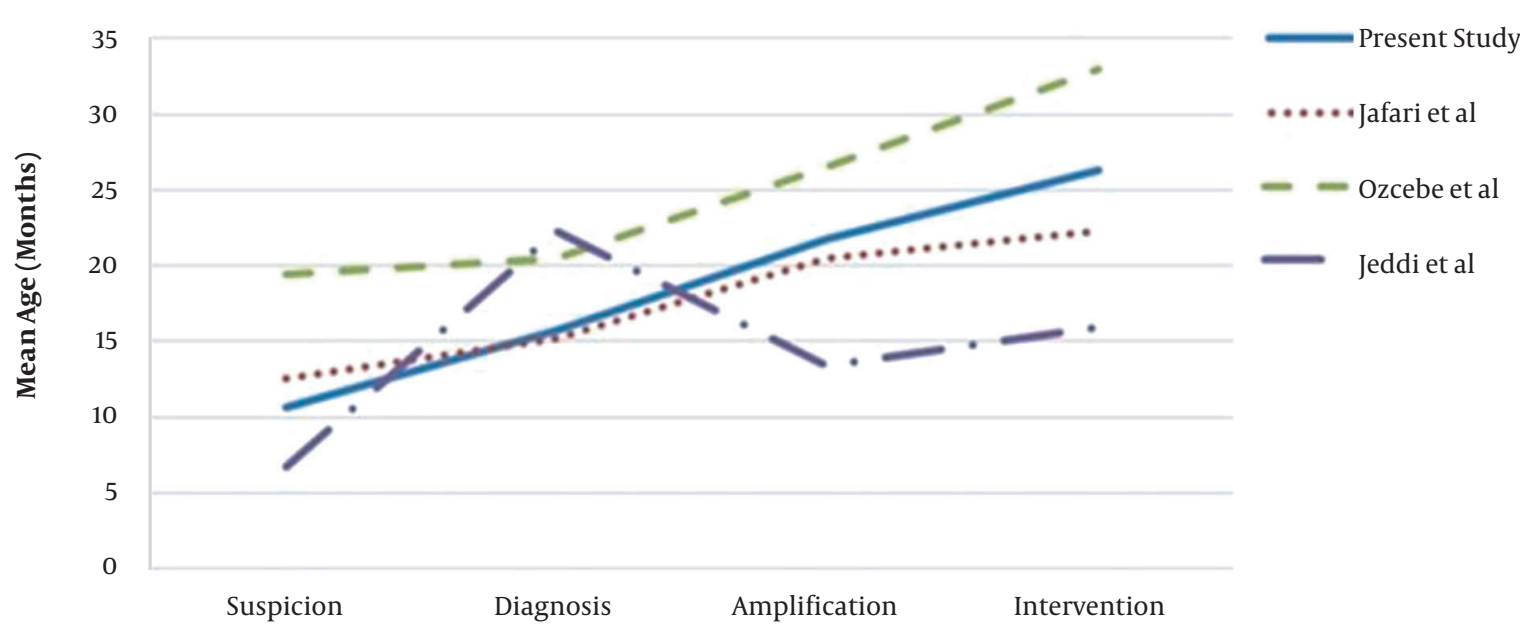

Figure 1. Comparison of the ages of children at hesitancy, recognition, reinforcement, and interposition among different studies

\begin{tabular}{|c|c|c|c|c|}
\hline \multirow{2}{*}{ Study } & \multicolumn{4}{|c|}{ Age (mo) } \\
\hline & Hesitancy & Recognition & Reinforcement & Interposition \\
\hline Present study & $10.73 \pm 7.15$ & $15.75 \pm 9.45$ & $21.81 \pm 8.19$ & $26.32 \pm 9.21$ \\
\hline Jafari et al. (17) & $12.6 \pm 8.9$ & $15.2 \pm 9.3$ & $20.5 \pm 11.1$ & $22.3 \pm 11.6$ \\
\hline Ozcebe et al. (14) & $12.5 \pm 7.9$ & $19.4 \pm 14.8$ & $26.5 \pm 14.8$ & $33 \pm 20$ \\
\hline Lotfi and Jafari (19) & $19.4 \pm 15.1$ & $25.9 \pm 16.8$ & $34.8 .5 \pm 21.3$ & $43.6 \pm 24.5$ \\
\hline Jeddi et al. (11) & $6.73 \pm 5.79$ & $9.35 \pm 5.79$ & $13.41 \pm 6.10$ & $41.25 \pm 11.12$ \\
\hline Prendergast et al. (16) & 8.16 & 14.58 & 19.05 & - \\
\hline
\end{tabular}

the chance to go to regular schools alongside their hearing peers. Currently, it is strongly intended to perform cochlear implantation at very young ages because this period is critical to learning. Delay in identifying severe to deep childhood hearing loss may lead to losing the best periods for learning a language. In turn, this limits educational achievement, decreases occupational and social opportunities, and it upsets and worries the families of hearing-impaired children (20-24).

Our research results show that there is no significant difference between high or not-high-risk and children at risk ages of hesitancy, recognition, reinforcement, and interposition. Jafari et al. (17), Kittrell and Arjmand (18), and Harrison and Roush (25) studies reported similar results and, in this regard, confirmed the findings of our research.

The main goal of neonatal hearing screening programs is to start the intervention at an early age, which can reduce the negative effects of hearing loss in children (< 6 months). On the other hand, it should be noted that early screening only helps to diagnose the disease quickly, achieving normal language and speech development is very difficult unless there is initial reinforcement and early 
education at the right age (26). The JCIH suggested general hearing screening in the 1th month, recognition of HL by three, and enrolment in primary interposition by six months of age (Guidelines 1-3-6) (27, 28).

\subsection{Conclusions}

The results indicated that the mean ages of HL hesitancy, recognition, reinforcement, and interposition were hopeless compared to the previous studies in Iran and other middle-east countries. However, there is still a noticeable gap between our results and the mean ages reported by the Joint Committee on Infant Hearing (JCIH) statement. Therefore, conducting a comprehensive protocol is highly recommended for primary recognition of $\mathrm{HL}$ and interposition.

\section{Acknowledgments}

This paper was extracted from the research dissertation with the registration Number HRC-9712. The authors of the present study expressed their gratitude and appreciation to those who helped them in this study. It was financially supported by the Vice-chancellor for Research and Hearing Research Center, AJUMS, Ahvaz, Iran.

\section{Footnotes}

Authors' Contribution: Study concept and design: Arash Bayat and Nader Saki. Acquisition of data: Amir Hossein Asadollah Pour and Hossein Bagheripour. Analysis and interpretation of data: Hasan Abshirini and Soheila Nikakhlagh. Drafting of the manuscript: Hossein Bagheripour.

Conflict of Interests: The authors do not have any conflicts of interest to declare.

Ethical Approval: The protocol of the current research was approved by the local Ethics Committee (Registration Number: IR.AJUMS.REC. 1397.958).

Funding/Support: This research was financially supported by the Vice-chancellor for Research of the AJUMS and Hearing Research Center, AJUMS, Ahvaz, Iran.

Informed Consent: The records from this study have been kept as confidential as possible. No individual identities have been used in any reports or publications resulting from the study. All data and retention methods ie, questionnaires, tapes, transcripts, summaries have given codes and stored separately from any names or other direct identification of the participants. Research information will be kept in locked files at all times. Only research personnel have had access to the files, and only those with an essential need to see names or other identifying information have had access to that particular file.

\section{References}

1. Nikakhlagh S, Yadollahpour A, Karimi M, Bagheripour H, Hematipour $\mathrm{S}$, Malehi AS, et al. Investigating Gender Differences on the Age of Suspicion of Children with Hearing Loss in Iran. Int J Ment Health Addict. 2016;15(2):271-6. doi: 10.1007/s11469-016-9671-5.

2. Jiang Y, Gu P, Li B, Gao X, Sun B, Song Y, et al. Analysis and Management of Complications in a Cohort of 1,065 Minimally Invasive Cochlear Implantations. Otol Neurotol. 2017;38(3):347-51. doi: 10.1097/MAO.0000000000001302. [PubMed: 28192378]. [PubMed Central: PMC5305286].

3. Szyfter W, Wróbel MJ, Szyfter-Harris J, Greczka G. Hearing Impairment in Polish Infants. Epidemiology. 2013;24(2):333. doi: 10.1097/EDE.0b013e31827b23a3.

4. Soleymani M, Nikakhlagh S, Hafezi G, Albokordi M, Saki N. Comparison of communication and social skills abilities of children with hearing impairment after cochlear implantation and parental expectations: A study conducted in Khuzestan cochlear implant center. Int J Pharm Technol. 2016;8:14791-802.

5. Ching TYC, Dillon H, Button L, Seeto M, Van Buynder P, Marnane V, et al. Age at Intervention for Permanent Hearing Loss and 5-Year Language Outcomes. Pediatrics. 2017;140(3):42-74. doi: 10.1542/peds.20164274. [PubMed: 28864712]. [PubMed Central: PMC5574730].

6. Saki N, Yadollahpour A, Moniri S, Karimi M, Bayat A, Abshirini H, et al. Investigating the impacts of cochlear implantation on the happiness and self-esteem of mothers of children with severe hearing loss. Int J Ment Health Addict. 2017;15(2):288-94.

7. Dashti R, Khiavi FF, Sameni SJ, Bayat A. Satisfaction with Hearing Aids among Aged Patients with Different Degrees of Hearing Loss and Length of Daily Use. J Audiol Otol. 2015;19(1):14-9. doi: 10.7874/jao.2015.19.1.14. [PubMed: 26185786]. [PubMed Central: PMC4491946].

8. Yoshinaga-Itano C, Alpiner JG, McCarthy PA. Assessment and intervention with preschool children who are deaf and hard of hearing. In: Alpiner J, McCarthy P, editors. Rehabilitative audiology: Children and adults. 3rd ed. Lippincott Williams \& Wilkins; 2000. p.140-77.

9. Yoshinaga-Itano C, Apuzzo ML. Identification of hearing loss after age 18 months is not early enough. Am Ann Deaf. 1998;143(5):380-7. doi: 10.1353/aad.2012.0151. [PubMed: 9893323].

10. Kim LS, Jeong SW, Lee YM, Kim JS. Cochlear implantation in children. Auris Nasus Larynx. 2010;37(1):6-17. doi: 10.1016/j.anl.2009.09.011. [PubMed: 19897328].

11. Jeddi Z, Jafari Z, Motasaddi Zarandy M. Effects of parents' level of education and economic status on the age at cochlear implantation in children. Iran J Otorhinolaryngol. 2012;24(66):7.

12. Butler IR, Basson S, Britz E, de Wet R, Korsten GB, Joubert G. Age of diagnosis of congenital hearing loss at Universitas Hospital, Bloemfontein. S Afr Med J. 2013;103(7):474-5. doi: 10.7196/samj.6395. [PubMed: 23802212].

13. Watkin $P$, Baldwin M. The longitudinal follow up of a universal neonatal hearing screen: the implications for confirming deafness in childhood. Int J Audiol. 2012;51(7):519-28. doi:10.3109/14992027.2012.673237. [PubMed: 22686437].

14. Ozcebe E, Sevinc S, Belgin E. The ages of suspicion, identification, amplification and intervention in children with hearing loss. Int J Pediatr Otorhinolaryngol.2005;69(8):1081-7. doi: 10.1016/j.ijporl.2005.03.002.

15. Kennedy CR, McCann DC, Campbell MJ, Law CM, Mullee M, Petrou $S$, et al. Language ability after early detection of permanent childhood hearing impairment. $N$ Engl J Med. 2006;354(20):2131-41. doi: 10.1056/NEJMoa054915. [PubMed: 16707750]. 
16. Prendergast SG, Lartz MN, Fiedler BC. Ages of diagnosis, amplification, and early intervention of infants and young children with hearing loss: findings from parent interviews. Am Ann Deaf. 2002;147(1):24-30. doi: 10.1353/aad.2012.0198. [PubMed: 12061188].

17. Jafari Z, Malayeri S, Ashayeri H. The ages of suspicion, diagnosis, amplification, and intervention in deaf children. Int J Pediatr Otorhinolaryngol. 2007;71(1):35-40. doi: 10.1016/j.ijporl.2006.08.014. [PubMed: 16997387].

18. Kittrell AP, Arjmand EM. The age of diagnosis of sensorineural hearing impairment in children. Int J Pediatr Otorhinolaryngol. 1997;40(23):97-106. doi:10.1016/s0165-5876(97)01506-1.

19. Lotfi Y, Jafari Z. Age of Suspicion, identification, hearing aid fitting and beginning to use aural rehabilitation services among Iranian children. Daneshvar J. 2002;48:39-44.

20. Saki N, Bayat A, Nikakhlagh S, Karimi M, Nikafrooz M, Daneshi A. A national Iranian cochlear implant registry (ICIR): Cochlear implanted recipient observational study. Int Tinnitus J. 2019;23(1):74-8.

21. Kos MI, Deriaz M, Guyot JP, Pelizzone M. What can be expected from a late cochlear implantation? Int J Pediatr Otorhinolaryngol. 2009;73(2):189-93. doi: 10.1016/j.ijporl.2008.10.009. [PubMed: 19054582].

22. Sarafraz M, Heidari M, Bayat A, Hanafi MG, Fahimi A, Farasat M, et al. Role of HRCT imaging in predicting the visibility of Round window (RW) on patients underwent cochlear implant surgery. Clin Epidemiol Glob Health. 2020;8(2):432-6. doi:10.1016/j.cegh.2019.10.003.

23. Liu Y, Dong R, Li Y, Xu T, Li Y, Chen X, et al. Effect of age at cochlear implantation on auditory and speech development of children with auditory neuropathy spectrum disorder. Auris Nasus Larynx. 2014;41(6):502-6. doi: 10.1016/j.anl.2014.06.001. [PubMed: 25194855].

24. Svirsky MA, Teoh SW, Neuburger H. Development of language and speech perception in congenitally, profoundly deaf children as a function of age at cochlear implantation. Audiol Neurootol. 2004;9(4):224-33. doi: 10.1159/000078392. [PubMed: 15205550].

25. Harrison M, Roush J. Age of suspicion, identification, and intervention for infants and young children with hearing loss: a national study. Ear Hear. 1996;17(1):55-62. doi: 10.1097/00003446-19960200000007. [PubMed: 8741968].

26. Moeller MP, Carr G, Seaver L, Stredler-Brown A, Holzinger D. Best practices in family-centered early intervention for children who are deaf or hard of hearing: an international consensus statement.J Deaf Stud Deaf Educ. 2013;18(4):429-45. doi: 10.1093/deafed/ent034. [PubMed: 24030911].

27. Muse C, Harrison J, Yoshinaga-Itano C, Grimes A, Brookhouser PE, Epstein S, et al. Supplement to the JCIH 2007 position statement: principles and guidelines for early intervention after confirmation that a child is deaf or hard of hearing. Pediatrics. 2013;131(4):e1324-49. doi: 10.1542/peds.2013-0008. [PubMed: 23530178].

28. American Academy of Pediatrics Joint Committee on Infant Hearing. Year 2007 position statement: Principles and guidelines for early hearing detection and intervention programs. Pediatrics. 2007;120(4):898-921. doi: 10.1542/peds.2007-2333. [PubMed: 17908777]. 